Original Article

\title{
Evaluation of age-related changes in lumbar facet joints using T2 mapping
}

\author{
Shinpei Enokida a, *, Shinji Tanishima a, Atsushi Tanida ${ }^{a}$, Tokumitsu Mihara ${ }^{a}$, \\ Chikako Takeda ${ }^{\mathrm{a}}$, Eijiro Yamashita ${ }^{\mathrm{b}}$, Hideki Nagashima ${ }^{\mathrm{a}}$ \\ a Department of Orthopedic Surgery, Faculty of Medicine, Tottori University, Yonago, Japan \\ ${ }^{\mathrm{b}}$ Division of Clinical Radiology, Tottori University Hospital, Yonago, Japan
}

\section{A R T I C L E I N F O}

\section{Article history:}

Received 8 August 2018

Received in revised form

9 January 2019

Accepted 14 February 2019

Available online xxx

\begin{abstract}
A B S T R A C T
Background: The purpose in this study is to investigate the T2 value of lumbar facet joint (FJ) in subjects without lumbar spinal disorders, age from 20s to 70s, using T2 mapping, and to evaluate the correlation between age and T2 value. And also, we investigated the T2 value of lumbar intervertebral disc (IVD) in the same way as FJ, and evaluated the correlation between the T2 value of FJ and that of IVD.

Methods: We investigated 60 volunteers (30 male, 30 female), who were recruited from six age groups, $20 \mathrm{~s}-70$ s ( 10 subjects in each decade; 5 male, 5 female). We measured the T2 values of FJ at the L4/5 level in axial image and those of IVD (nucleus pulposus; NP, anterior and posterior annulus fibrosus; AAF and $\mathrm{PAF}$ ) at the $\mathrm{L} 4 / 5$ level in midline sagittal image. We investigated the correlation between age and $\mathrm{T} 2$ value of FJ, and the correlation between the T2 value of FJ and that of IVD.

Results: There was a strong positive correlation between age and T2 value of $\mathrm{FJ}(\mathrm{r}=0.717)$. Age and T2 values of IVD were negatively correlated (NP; $r=-0.728$, AAF; $r=-0.696$, PAF; $r=-0.580$ ). There was a negative correlation between T2 value of FJ and that of IVD (NP; $r=-0.575$, AAF; $r=-0.617$, PAF; $\mathrm{r}=-0.492$ ).

Conclusions: T2 value of FJ was significantly increased as age rose. Our results suggest that T2 mapping could detect the degenerative changes of FJ related to aging even in subjects without lumbar spinal disorders. The results of this study will be the reference data of FJ T2 value in order to evaluate the relationship between low back pain and FJ using T2 mapping.
\end{abstract}

๑ 2019 The Japanese Orthopaedic Association. Published by Elsevier B.V. All rights reserved.

\section{Introduction}

Low back pain (LBP) is one of the most frequent symptoms [1,2], and so there are a lot of opportunities to examine patients with LBP in clinic. The number of patients suffering from LBP is very high all over the world, and so LBP is a serious problem affecting medical economy $[1,3]$. According to the data from comprehensive survey of living conditions, 2013 in Japan, which has been performed by Ministry of Health, Labor and Welfare in our country, the complaining ratio of the LBP was approximately $9.2 \%$ in male (92.2 per 1000 people), and $11.8 \%$ in female ( 118.2 per 1000 people) [4]. In the past, many research on causes of LBP have been conducted, and it is

\footnotetext{
* Corresponding author. Department of Orthopedic Surgery, Faculty of Medicine, Tottori University, 36-1 Nishi-cho, Yonago, Tottori, 683-8504, Japan. Fax: +81859 386589.

E-mail address: enoshin@med.tottori-u.ac.jp (S. Enokida).
}

widely accepted that degeneration of the lumbar facet joint (FJ) is one of the main causes of LBP [1-3,5,6]. However, unclear points remain for details about the pathology of FJ.

To evaluate degeneration of FJ, magnetic resonance imaging (MRI) is an indispensable modality. However, it is difficult to quantitative assessment using MRI in conventional imaging procedure [7]. In recent years, improvement of MRI led to the development of quantitative imaging techniques [8-11]. T2 mapping is one of useful method of MRI for clinical quantitative imaging of articular cartilage $[1,12,13]$. Most of the previous reports on T2 mapping in limb joints studied large joints such as knees $[10,11,14,15]$. On the other hand, as concerns with the field of spine, studies on lumbar intervertebral disc (IVD) accounted for much $[5,6,16-20]$. Assessment of T2 values of degenerated IVDs and relevance of LBP to T2 values of IVDs were reported in several peerreviewed literature $[5,6]$. But, there are few reports on the research of FJ using T2 mapping $[1,12,13]$. Furthermore, as far as we know, 
there is no report that $\mathrm{T} 2$ values of FJ in subjects without lumbar spinal disorders, who were divided into age groups, were investigated using T2 mapping. In order to conduct research to evaluate the relationship between LBP and FJ using T2 mapping, we consider that it is necessary to investigate $\mathrm{T} 2$ values of $\mathrm{FJ}$ in subjects without lumbar spinal disorders as reference $\mathrm{T} 2$ values before that.

Therefore, the purposes of our study were to investigate the T2 value of lumbar FJ in volunteers using T2 mapping, and to evaluate the correlation between age and $\mathrm{T} 2$ value. We consider that the T2 value of lumbar FJ in volunteers will be the reference data of FJ T2 value for further research of FJ using T2 mapping. And also, in order to compare with FJ, we investigated the T2 value of lumbar IVD in the same way as FJ, and evaluated the correlation between the T2 value of FJ and that of IVD.

\section{Materials and methods}

\subsection{Subjects}

This study was approved by our institutional review board, and written informed consent was obtained from each subject before enrollment in the study. We posted flyers about this study in our hospital to recruit volunteers. The exclusion criteria were: 1) history of diagnosis of lumbar spine disease, 2) previous surgical history for spinal disease, 3 ) low back pain at the time of recruitment with Numerical Rating Scale (NRS) > 3,4) rheumatoid arthritis or other inflammatory disease, 5) infection, 6) neoplastic disease, 7) remarkable lumbar spine degeneration, 8) contraindications for MRI, or 9) during pregnancy. Sixty participants (30 male, 30 female) were included. Volunteers were recruited from six age groups: $20-29$ years (20s); 30-39 years (30s); 40-49 years (40s); 50-59 years (50s); 60-69 years (60s); and 70-79 years (70s). Each group had 10 volunteers ( 5 men, 5 women). Among these volunteers, there were just normal subjects and patients as well. No patients were diagnosed with lumbar spine disease in volunteers. Seven male volunteers in 50s-70s were patients with rotator cuff injury, and one male volunteer in 60s was patient with finger tendon injury. Other volunteers are just normal subjects. The definition of remarkable lumbar spine degeneration is grade 2 and 3 of MRI grading system of Weishaupt, which is criteria for grading osteoarthritis of FJ. In this study, volunteers whose FJ with grade 2 and 3 were not included. In this study, the definition of subjects without lumbar spinal disorders was that there were no history of diagnosis of lumbar spine disease and treatment, and there were no neurological symptoms and LBP with NRS $>3$. We regarded the subjects who answered "yes" against this question "Do you feel low back pain (LBP) with NRS > 3 for 3 months your daily life?" were subjects LBP. We excluded these subjects. All volunteers underwent MRI of the lumbar spine, and no volunteers interrupted MRI for feeling ill.

\subsection{Ethical approval}

All procedures performed in studies involving human participants were in accordance with the ethical standards of the institutional and/or national research committee and with the 1964 Helsinki declaration and its later amendments or comparable ethical standards.

\subsection{MRI protocol and image analysis}

MR imaging was performed with a 3.0 T MR unit (MAGNETOM Skyra; Siemens, Erlangen, Germany) that had a gradient strength of $45 \mathrm{mT} / \mathrm{m}$ and with use of a phased-array spine coil. The imaging protocol consisted of axial and sagittal planes, multi-echo spin- echo T2-weighted sequence performed with the following parameters: repetition time msec/echo time msec, 1500/15, 30, 45, 60, 75, and 90; field of view, $200 \times 200 \mathrm{~mm}$; pixel matrix, $128 \times 128$; voxel size, $1.6 \times 1.6 \times 3.0 \mathrm{~mm}$; bandwidth, $200 \mathrm{~Hz} /$ pixel; one signal acquired; six sections; and total acquisition time, 3 min $54 \mathrm{~s}$. T2 relaxation times were obtained from online reconstructed T2 maps by using a pixelwise, monoexponential nonnegative least-squares fit analysis (MapIt; Siemens Medical Solutions, Erlangen, Germany) [15].

We obtained axial and sagittal T2-weighted cross-sectional images of L4/5 level. Of axial images, imaging cut sequences were at least $3 \mathrm{~mm}$, using the way described by Williams et al. [21], and we selected one demonstrating most closely bisected the FJs at L4/5 level. Of sagittal images, we selected a midline sagittal image at L4/ 5 level. From selected axial and sagittal images, T2 map images were created. The regions of interest (ROIs) were set manually by an author (SE). ROIs for FJs were drawn on axial first echo image across the area including the joint space (Fig. 1A), and copied onto the T2 map images (Fig. 1B), and so T2 values of FJs were measured automatically. To measure T2 values of IVD, we used the way described by Trattnig et al. [22] and Takashima et al. [19]. In sagittal view, IVD at L4/5 was divided into five equal areas, designating the front fifth of the anterior annulus fibrosus (AAF), the middle fifth of the nucleus pulposus (NP), and the last fifth of the posterior annulus fibrosus (PAF). ROIs for IVDs were drawn on sagittal first echo image across the area including AAF, NP and PAF (Fig. 2A), and copied onto the T2 map images (Fig. 2B), and so T2 values of IVDs were measured automatically. We measured the T2 values of FJs for both right and left sides at the L4/5 level in axial image and those of IVD (AAF, NP and PAF) at the L4/5 level in midline sagittal image.

\subsection{Statistical analysis}

The statistical evaluation was performed using Statcel 4 (Statcel - The Useful Addin Forms on Excel - 4th ed.). We investigated the following items as the background of volunteers, 1) body height, 2) body weight, 3) body mass index (BMI), 4) NRS of LBP. Subsequently, we also statistically investigated the correlation between age and T2 value of FJ, between age and T2 value of IVD, and then, between T2 value of FJ and that of IVD using Pearson's rankcorrelation coefficient. We calculated the mean T2 value of the right and left FJs, and used the value for our study. The strength of association was determined by considering the correlation coefficients and their significance. A correlation was considered very strong if there was an absolute value of $0.80-1.00$, a strong correlation by $0.60-0.79$, a moderate correlation by $0.40-0.59$, a weak correlation by $0.20-0.39$, and no correlation by an absolute value under 0.20 . A p-value of less than 0.05 was considered statistically significant.

\section{Results}

\subsection{Demographic volunteers data}

The mean age was 48.8 years (23-79 years), and by age grouping, 26.2, 32.7, 42.0, 54.6, 64.0, 74.0 years in 20s-70s, respectively. There was no statistically significant correlation between age and body height $(r=-0.230, P=0.077)$ (Fig. $3 \mathrm{~A})$, body weight $(\mathrm{r}=-0.016, \mathrm{P}=0.906)$ (Fig. $3 \mathrm{~B})$, body mass index (BMI) $(r=0.207, P=0.113)$ (Fig. $3 C)$, or NRS of $\operatorname{LBP}(r=-0.189, P=0.147)$.

\subsection{T2 values of lumbar FJ and IVD}

Table 1 shows T2 values of lumbar FJ and IVD (AAF, NP, PAF). T2 values are given as mean \pm standard deviation. We calculated the 

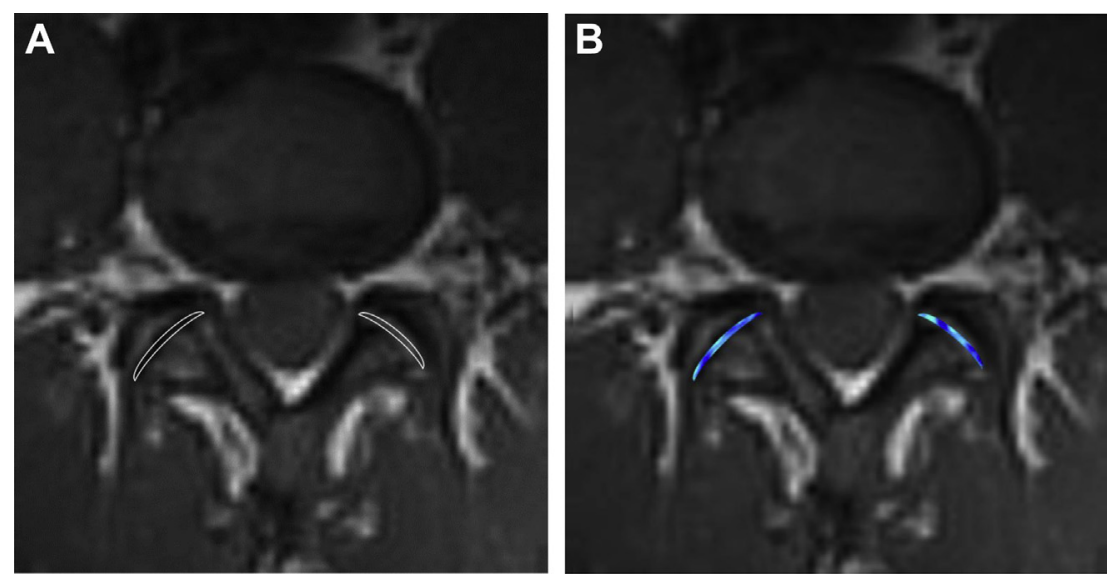

Fig. 1. The regions of interest (ROIs) for lumbar facet joints (FJs) at L4/5 were drawn on axial first echo image across the area including the bilateral joint space (A), and copied onto the T2 map images (B).

mean T2 value of the right and left FJs, and used the value for our study.

\subsection{Correlation between age and $T 2$ value of FJ}

There was a strong positive correlation between age and T2 value of $\mathrm{FJ}(\mathrm{r}=0.717, \mathrm{P}<0.0001)$ (Fig. 4). T2 value of $\mathrm{FJ}$ was significantly increased as age rose.

\subsection{Correlation between age and T2 value of IVD (AAF, NP, PAF)}

There was a strong negative correlation between age and T2 value of AAF $(r=-0.728, P<0.0001)$ (Fig. $5 A), N P(r=-0.696$, $\mathrm{P}<0.0001$ ) (Fig. 5B), and a moderate negative correlation between age and T2 value of PAF $(r=-0.580, P<0.0001)$ (Fig. $5 C$ ). T2 value of IVD was significantly decreased as age rose.

\subsection{Correlation between T2 value of FJ and that of IVD (AAF, NP, $P A F)$}

There was a strong negative correlation between $\mathrm{T} 2$ value of $\mathrm{FJ}$ and that of AAF $(r=-0.617, \mathrm{P}<0.0001)$ (Fig. $6 \mathrm{~A})$, and a moderate negative correlation between $\mathrm{T} 2$ value of $\mathrm{FJ}$ and that of $\mathrm{NP}(\mathrm{r}=-0.575$, $\mathrm{P}<0.0001$ ) (Fig. 6B), PAF $(\mathrm{r}=-0.492, \mathrm{P}<0.0001)$ (Fig. 6C). T2 value of FJ was significantly increased as T2 value of IVD decreased.

\subsection{Correlation between NRS of LBP and T2 value of FJ and IVD} (AAF, NP, PAF)

There was no statistically significant correlation between NRS of LBP and T2 value of $\mathrm{FJ}(\mathrm{r}=0.067, \mathrm{P}=0.612)$. On the other hand, there was a weak negative correlation between NRS of LBP and T2 value of IVD-AAF $(r=-0.291, P=0.024)$, IVD-NP $(r=-0.381$, $\mathrm{P}=0.003)$, and IVD-PAF $(\mathrm{r}=-0.389, \mathrm{P}=0.002)$.

\section{Discussion}

T2 mapping is one of useful modalities for clinical quantitative imaging of articular cartilage and IVD [1,12]. It allows quantification of water content and collagen orientation by measuring $\mathrm{T} 2$ values, and can be used to detect degeneration of tissue in cartilage and IVDs occurring in the early stage [8,9]. T2 values are dependent on the integrity of collagen sequence, and the quantity of water and proteoglycan content $[8,9]$.

The FJ is a synovial joint, and so the joint surface is covered by hyaline cartilage and has a joint capsule $[2,23]$. The structure is similar to joints of limbs, such as the knee joint, and also osteoarthritis of the FJ is similar to that of joints of limbs [2,23]. Therefore, it is predicted that changes in $\mathrm{T} 2$ values with degeneration of FJs would be similar to those with degeneration of cartilage in knee joints $[12,13]$. The previous studies on T2 mapping regarding knee joint
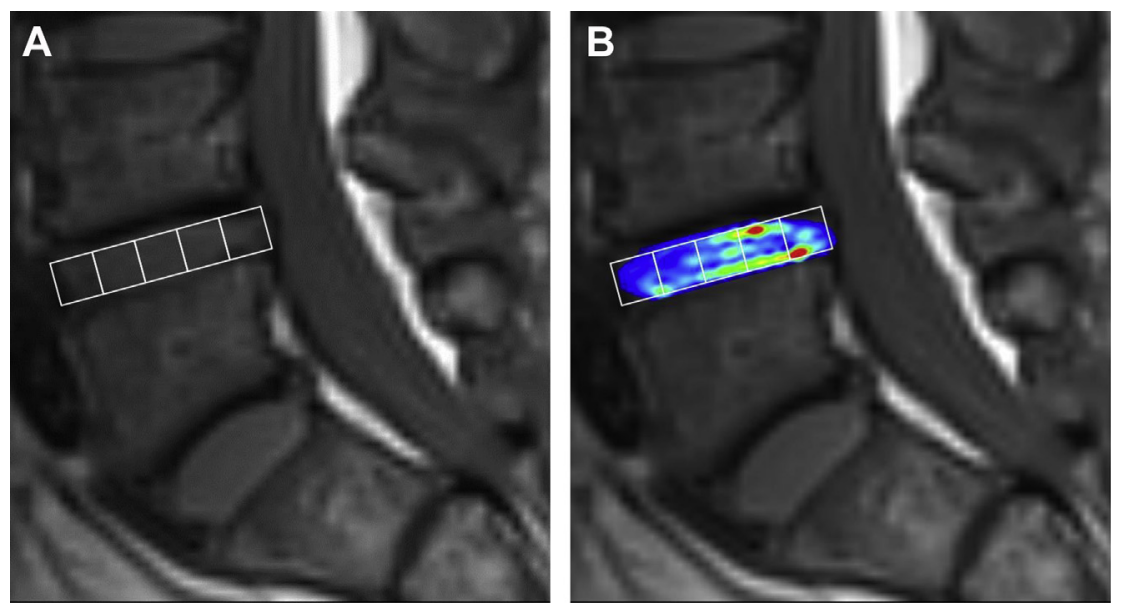

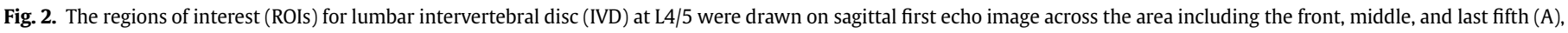
and copied onto the T2 map images (B). 
demonstrated higher T2 values in degenerative cartilage compared to healthy knee joints [14]. Compositional changes in water content and collagen sequence of the matrix of cartilage can be evaluated quantitatively using T2 mapping. T2 values reflect the integrity of collagen sequence, the quantity of water and proteoglycan content, and the mobility of protons in water molecules in collagen matrix $[8,9]$. Therefore, T2 values of degenerative cartilage increase due to the collapse of collagen sequence, the increase in water content, and increased mobility of protons in water molecules in collagen matrix

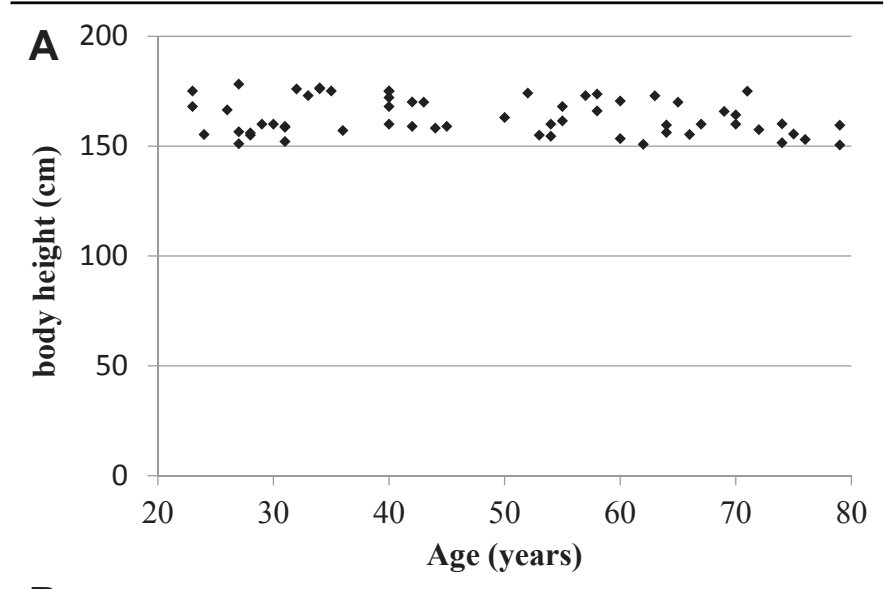

B

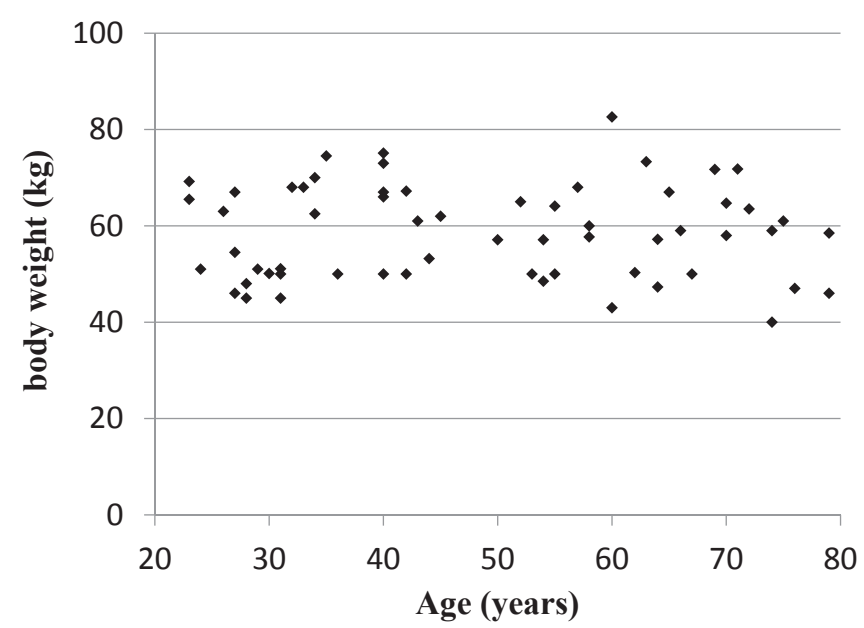

C

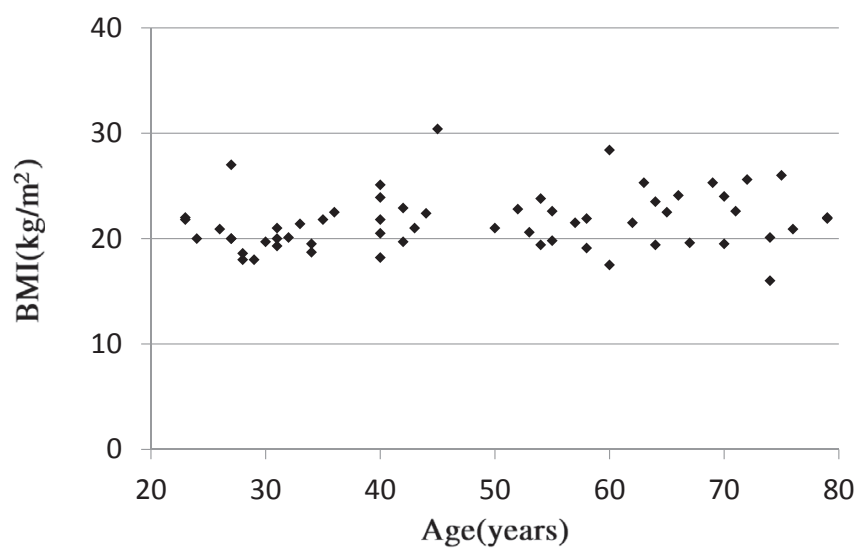

Fig. 3. The correlation between age and body height (A), body weight (B), body mass index (BMI) (C).
$[8,9,12]$. On the other hand, T2 values of normal cartilage do not increase because the mobility of protons is restricted due to the dense and regular collagen matrix.

Takashima et al. [13] evaluated lumbar FJ in group with lumbar degenerative spondylolisthesis (DS) and non-spondylolisthesis (NS) by T2 mapping, and reported that T2 values of FJ were significantly higher in the DS than NS group. They considered that water content increased as a results of degeneration in the FJ and that T2 value increased significantly in the DS group. Stelzeneder et al. [12] evaluated the relationship between LBP and T2 value of FJ by using T2 mapping. They compared 10 patients (the mean age was 31.5, range 20-50) suffering from acute or chronic LBP and 5 asymptomatic volunteers (the mean age was 25.4 , range $23-28$ ). In their results, there was no difference in $\mathrm{T} 2$ value of $\mathrm{FJ}$ between patients with LBP and asymptomatic volunteers. They assessed the results that their subjects' age was relatively young, so degenerative changes of FJ did not advance. In another report [24], it was stated that FJs osteoarthritis is uncommon before the age of 45 years. Considering their discussion, we speculate that there is no difference in T2 value of FJ between patients with LBP and volunteers only for the young generation, but there will be a difference in $\mathrm{T} 2$ value of FJ for elderly generation. As far as we know, there is no study to investigate $\mathrm{T} 2$ values of $\mathrm{FJ}$ in elderly generation volunteers using T2 mapping. This study is a first article to investigate age-

Table 1

T2 values of lumbar facet joint and intervertebral disc.

\begin{tabular}{lllll}
\hline \multirow{2}{*}{ Age } & \multicolumn{4}{l}{ T2 values $(\mathrm{msec})$} \\
\cline { 2 - 5 } & FJ & IVD-AAF & IVD-NP & IVD-PAF \\
\hline $20 \mathrm{~s}$ & $79.5 \pm 8.2$ & $99.8 \pm 14.9$ & $171.2 \pm 47.4$ & $155.5 \pm 51.5$ \\
$30 \mathrm{~s}$ & $78.6 \pm 5.2$ & $99.6 \pm 28.3$ & $155.9 \pm 56.8$ & $145.9 \pm 76.5$ \\
$40 \mathrm{~s}$ & $91.6 \pm 11.5$ & $73.1 \pm 16.8$ & $106.1 \pm 39.9$ & $95.9 \pm 46.4$ \\
$50 \mathrm{~s}$ & $98.4 \pm 17.3$ & $62.9 \pm 16.9$ & $81.4 \pm 25.4$ & $80.1 \pm 33.1$ \\
$60 \mathrm{~s}$ & $109.3 \pm 12.0$ & $58.1 \pm 16.5$ & $72.7 \pm 19.5$ & $71.5 \pm 16.2$ \\
$70 \mathrm{~s}$ & $111.8 \pm 10.9$ & $50.2 \pm 8.5$ & $74.8 \pm 15.9$ & $71.9 \pm 18.4$ \\
\hline
\end{tabular}

$\mathrm{T} 2$ values are given as mean \pm standard deviation.

We calculated the mean $\mathrm{T} 2$ value of the right and left FJs, and used the value for our study.

FJ: Facet joint.

IVD: Intervertebral disc.

AAF: Anterior annulus fibrosus.

NP: Nucleus pulposus.

PAF: Posterior annulus fibrosus.

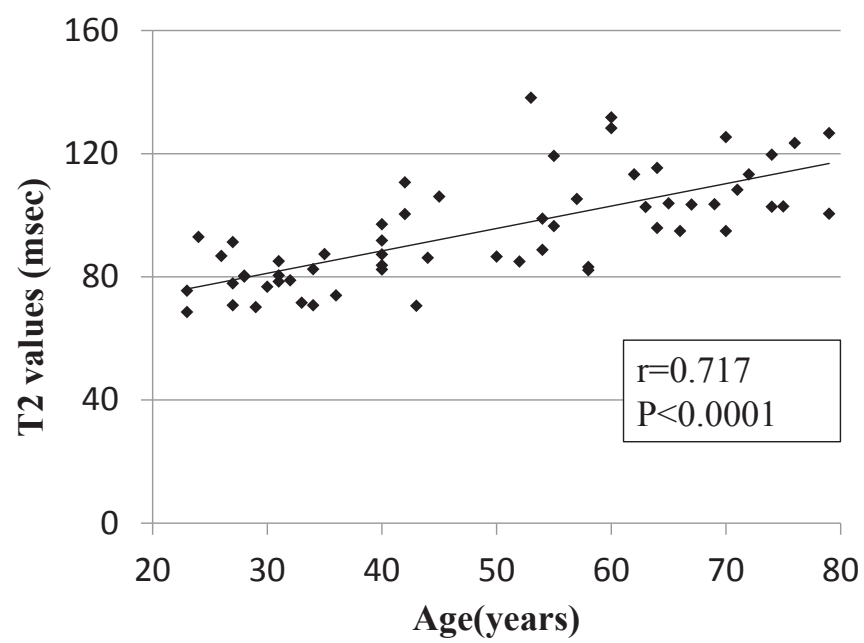

Fig. 4. The correlation between age and $\mathrm{T} 2$ value of lumbar facet joint (FJ). We calculated the mean $\mathrm{T} 2$ value of the right and left FJs, and used the value for our study. 


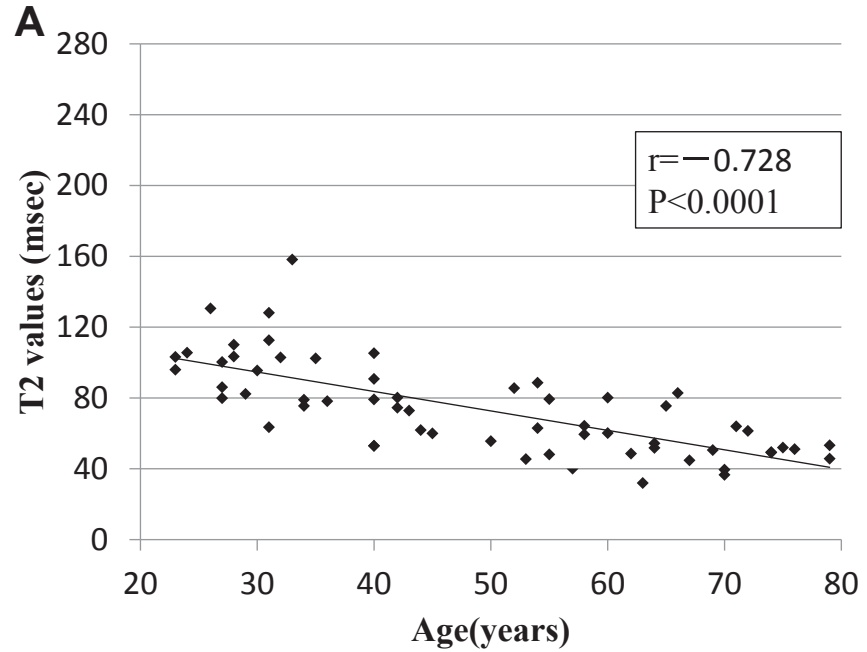

B

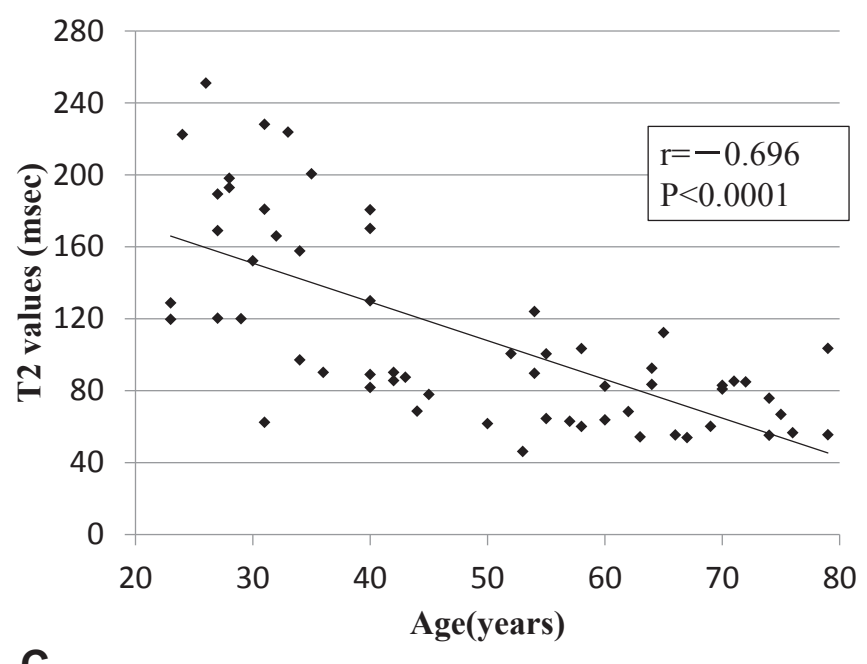

C

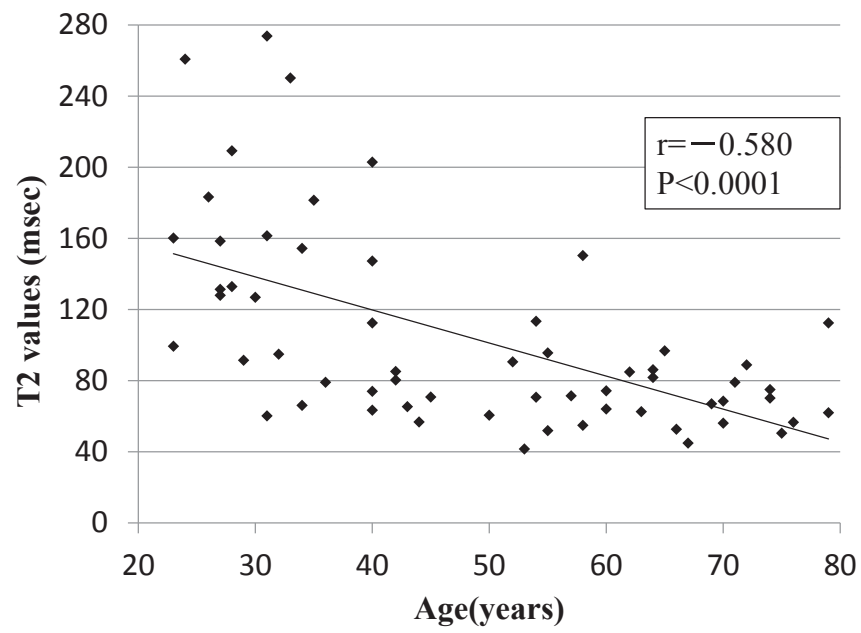

Fig. 5. The correlation between age and $T 2$ value of lumbar intervertebral disc (IVD)anterior annulus fibrosus (AAF) (A), nucleus pulposus (NP) (B), posterior annulus fibrosus (PAF) (C)

related changes in $\mathrm{T} 2$ value of $\mathrm{FJ}$ for volunteers from young to elderly generation using T2 mapping.

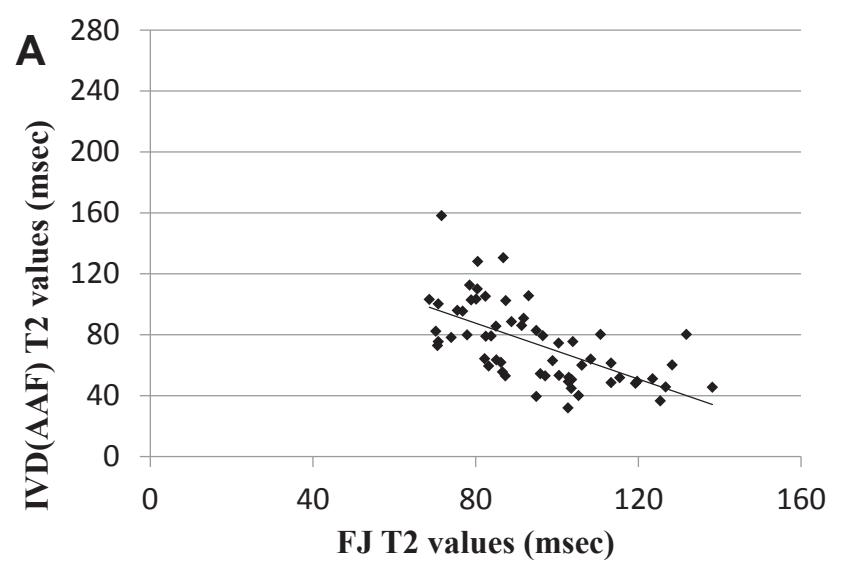

B
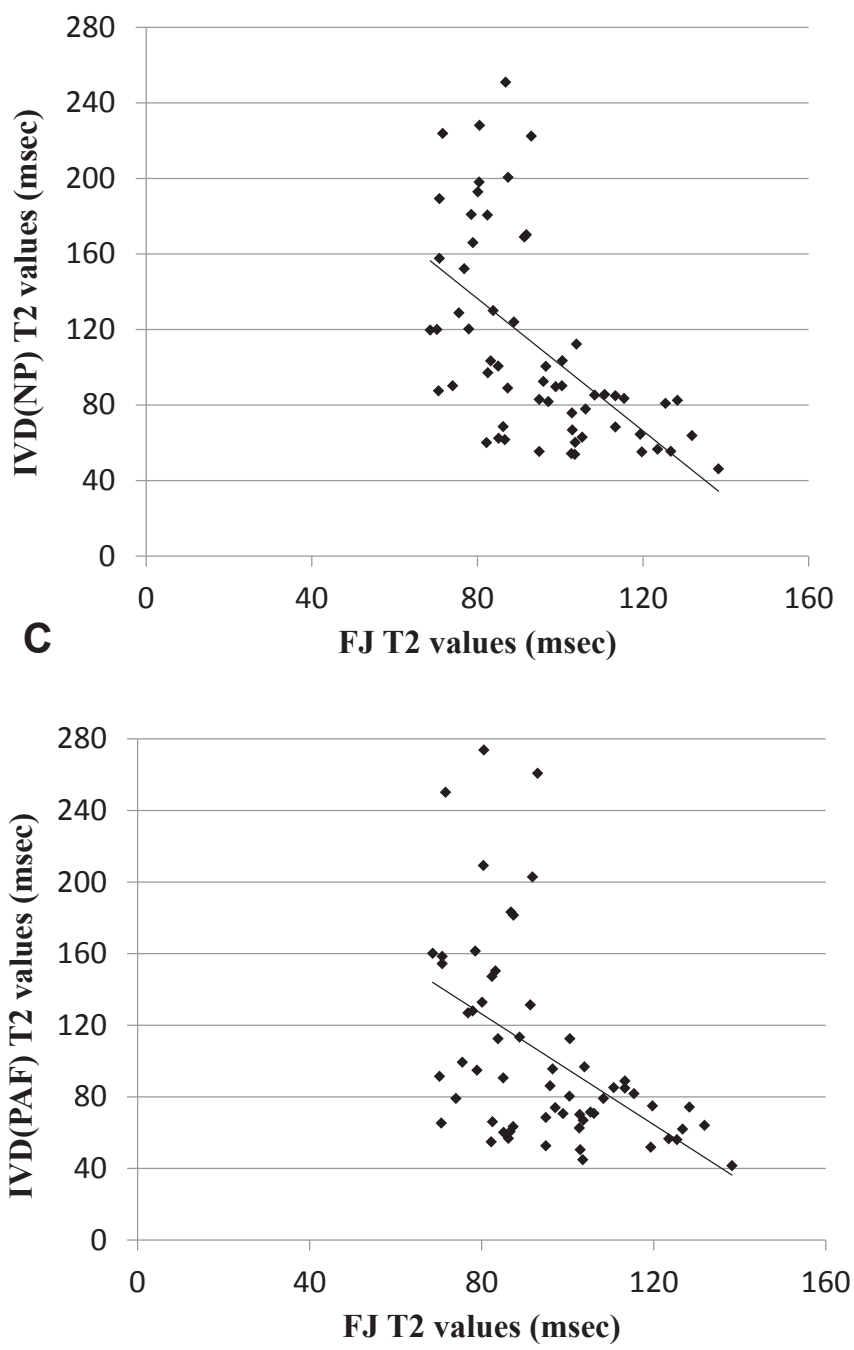

Fig. 6. The correlation Between $T 2$ Value of lumbar facet joint (FJ) and that of intervertebral disc (IVD)-anterior annulus fibrosus (AAF) (A), nucleus pulposus (NP) (B), posterior annulus fibrosus (PAF) (C).

Several studies on LBP and IVD using T2 mapping have been reported, but few studies on LBP and FJ using it have been reported. The report of Stelzeneder et al. is an only article to evaluate about the relationship between LBP and T2 value of FJ. We consider that T2 mapping is a useful method of MRI for evaluating LBP originating FJ, 
just as it is to evaluate LBP originating IVD. In order to evaluate the relationship between LBP and FJ using T2 mapping, it is necessary to investigate $\mathrm{T} 2$ values of $\mathrm{FJ}$ in normal lumbar vertebra as reference $\mathrm{T} 2$ values before that. In this study, we calculated T2 value of lumbar FJ in volunteers, age from 20s to 70s, using T2 mapping. We consider that the data of Table 1 will be the reference data of FJ T2 value for further research of FJ using T2 mapping. Because, as far as we know, there is no report that T2 values of $\mathrm{FJ}$ in subjects without lumbar spinal disorders, who were divided into age groups, were investigated using T2 mapping. To obtain the reference data of FJ T2 value is another object of this research. We estimate that this makes it possible to evaluate T2 value of patients with LBP in more detail.

The current study demonstrated a strong positive correlation between age and T2 value of FJ. T2 value of FJ was significantly increased as age rose. Our results suggest that $\mathrm{T} 2$ mapping could detect the degenerative changes of FJ related to aging even in subjects without lumbar spinal disorders. To our best knowledge, there has been no reports investigating the relationship between age and $\mathrm{T} 2$ value of FJ, and the age of the subject was not taken into consideration. Therefore, there are variations in T2 value of FJ among previous reports. We emphasize it important to compare with subjects with same age generation in the study of lumbar FJ or IVD using T2 mapping.

There are some limitations in this study. Firstly, the accuracy of calculation methods of $\mathrm{T} 2$ values was uncertain. ROIs were selected manually by an author, and it was difficult to draw ROIs if MRI image was poor resolution, especially articular surfaces of FJs. Secondly, the influence of facet effusion on T2 value of FJ is still controversial. The solution to the influence of facet effusion is not mentioned in other peer-reviewed literature. In this study, there were no volunteers who admitted facet effusion with axial T2weighted cross-sectional images of L4/5 level by chance. We consider that lumbar spine degeneration in the early stage without facet effusion is a good indication for T2 mapping. Thirdly, we did not evaluate the degree of degeneration of lumbar spine with plain radiographs and CT scan. We evaluated degeneration of FJ with only MRI. Because the subjects are volunteers, we did not obtain plain radiographs and $\mathrm{CT}$ scan, considering the risk of radiation exposure. And finally, we included subjects with no history of diagnosis of lumbar spine disease, symptoms and treatment in this study. In the future study, we will include patients with lumbar degeneration with symptoms such as LBP and lower extremity pain.

In conclusions, our results suggest that $\mathrm{T} 2$ mapping could detect the degenerative changes of FJ related to aging even in subjects without lumbar spinal disorders. The results of this study will be the reference data of FJ T2 value in order to evaluate the relationship between LBP and FJ using T2 mapping.

\section{Conflict of interest}

The authors declare that there is no conflict of interest.

\section{Acknowledgments}

This work was supported by the Grant-in-Aid for Scientific Research of Japan Society for the Promotion of Science, Grant Number 16K10820.

\section{References}

[1] Hu J, Zhang Y, Duan C, Peng X, Hu P, Lu H. Feasibility study for evaluating early lumbar facet joint degeneration using axial T1 rho, T2, and T2* mapping in cartilage. J Magn Reson Imag JMRI 2017 Aug;46(2):468-75.

[2] Weishaupt D, Zanetti M, Boos N, Hodler J. MR imaging and CT in osteoarthritis of the lumbar facet joints. Skeletal Radiol 1999 Apr;28(4):215-9.
[3] Dagenais S, Caro J, Haldeman S. A systematic review of low back pain cost of illness studies in the United States and internationally. Spine J - Offic J North Ame Spine Soc 2008 Jan-Feb;8(1):8-20.

[4] Comprehensive survey of living conditions. Ministry of Health, Labour and Welfare in Japan; 2014 Dec. Available from: http://www.mhlw.go.jp/toukei/ list/dl/20-21-h25.pdf. [Accessed 5 June 2017].

[5] Nilsson M, Lagerstrand K, Kasperska I, Brisby H, Hebelka H. Axial loading during MRI influences T2-mapping values of lumbar discs: a feasibility study on patients with low back pain. European spine journal : official publication of the European Spine Society. Eur Spinal Deformity Soc Eur Section Cervical Spine Res Soc 2016 Sep;25(9):2856-63.

[6] Ogon I, Takebayashi T, Takashima H, Tanimoto K, Ida K, Yoshimoto M, Fujiwara H, Kubo T, Yamashita T. Analysis of chronic low back pain with magnetic resonance imaging T2 mapping of lumbar intervertebral disc. J Orthop Sci 2015 Mar;20(2):295-301.

[7] Gellhorn AC, Katz JN, Suri P. Osteoarthritis of the spine: the facet joints. Nat Rev Rheumatol 2013 Apr;9(4):216-24.

[8] Blumenkrantz G, Majumdar S. Quantitative magnetic resonance imaging of articular cartilage in osteoarthritis. Eur Cells Mater 2007 May 15;15(13): 76-86.

[9] Braun HJ, Gold GE. Advanced MRI of articular cartilage. Imag Med 2011 Oct;3(5):541-55.

[10] Pai A, Li X, Majumdar S. A comparative study at 3 T of sequence dependence of T2 quantitation in the knee. Magn Reson Imag 2008 Nov;26(9):1215-20.

[11] Prasad AP, Nardo L, Schooler J, Joseph GB, Link TM. T(1)rho and T(2) relaxation times predict progression of knee osteoarthritis. Osteoarthritis Cartilage 2013 Jan;21(1):69-76.

[12] Stelzeneder D, Messner A, Vlychou M, Welsch GH, Scheurecker G, Goed S Pieber K, Pflueger V, Friedrich KM, Trattnig S. Quantitative in vivo MRI evaluation of lumbar facet joints and intervertebral discs using axial T2 mapping. Eur Radiol 2011 Nov;21(11):2388-95.

[13] Takashima H, Takebayashi T, Yoshimoto M, Terashima Y, Ida K, Shishido H Imamura R, Akatsuka Y, Shirase R, Fujiwara H, Kubo T, Yamashita T. Investigation of intervertebral disc and facet joint in lumbar spondylolisthesis using T2 mapping. Magn Reson Med Sci 2014 Oct;13(4):261-6.

[14] Caglar E, Sahin G, Ogur T, Aktas E. Quantitative evaluation of hyaline articular cartilage T2 maps of knee and determine the relationship of cartilage T2 values with age, gender, articular changes. Eur Rev Med Pharmacol Sci 2014 Nov;18(22):3386-93.

[15] Mamisch TC, Trattnig S, Quirbach S, Marlovits S, White LM, Welsch GH. Quantitative T2 mapping of knee cartilage: differentiation of healthy contro cartilage and cartilage repair tissue in the knee with unloading-initial results. Radiology 2010 Mar;254(3):818-26.

[16] Menezes-Reis R, Salmon CE, Bonugli GP, Mazoroski D, Tamashiro MH, Savarese LG, Nogueira-Barbosa MH. Lumbar intervertebral discs T2 relaxometry and T1rho relaxometry correlation with age in asymptomatic young adults. Quant Imag Med Surg 2016 Aug;6(4):402-12.

[17] Messner A, Stelzeneder D, Trattnig S, Welsch GH, Schinhan M, Apprich S, Brix M, Windhager R, Trattnig S. Does T2 mapping of the posterior annulus fibrosus indicate the presence of lumbar intervertebral disc herniation? A 3.0 Tesla magnetic resonance study. Eur Spine J - Offic Publ Eur Spine Soc Eur Spinal Deformity Soc Eur Section Cervical Spine Res Soc 2017 Mar;26(3): $877-83$.

[18] Nisolle JF, Bihin B, Kirschvink N, Neveu F, Clegg P, Dugdale A, Wang X, Vandeweerd JM. Prevalence of age-related changes in ovine lumbar intervertebral discs during computed tomography and magnetic resonance imaging. Comp Med 2016 Aug;66(4):300-7.

[19] Takashima H, Takebayashi T, Yoshimoto M, Terashima Y, Tsuda H, Ida K, Yamashita T. Correlation between T2 relaxation time and intervertebral disk degeneration. Skeletal Radiol 2012 Feb;41(2):163-7.

[20] Yoon MA, Hong SJ, Kang CH, Ahn KS, Kim BH. T1rho and T2 mapping of lumbar intervertebral disc: correlation with degeneration and morphologic changes in different disc regions. Magn Reson Imag 2016 Sep;34(7):932-9.

[21] Williams R, Cheung JP, Goss B, Rajasekaran S, Kawaguchi Y, Acharya S, Kawakami M, Satoh S, Chen WJ, Park CK, Lee CS, Foocharoen T, Nagashima H, Kuh S, Zheng Z, Condor R, Ito M, Iwasaki M, Jeong JH, Luk KD, Prijambodo B, Rege A, Jahng TA, Luo Z, Tassanawipas W, Acharya N, Pokharel R, Shen Y, Ito T, Zhang Z, Aithala PJ, Kumar GV, Jabir RA, Basu S, Li B, Moudgil V, Sham P, Samartzis D. An international multicenter study assessing the role of ethnicity on variation of lumbar facet joint orientation and the occurrence of degenerative spondylolisthesis in Asia Pacific: a study from the AOSpine Asia Pacific research collaboration consortium. Glob Spine J 2016 Feb;6(1):35-45.

[22] Trattnig S, Stelzeneder D, Goed S, Reissegger M, Mamisch TC, PaternostroSluga T, Weber M, Szomolanyi P, Welsch GH. Lumbar intervertebral disc abnormalities: comparison of quantitative T2 mapping with conventional MR at 3.0 T. Eur Radiol 2010 Nov;20(11):2715-22.

[23] Kalichman L, Hunter DJ. Lumbar facet joint osteoarthritis: a review. Semin Arthritis Rheum 2007 Oct;37(2):69-80.

[24] Fujiwara A, Tamai K, Yamato M, An HS, Yoshida H, Saotome K, Kurihashi A. The relationship between facet joint osteoarthritis and disc degeneration of the lumbar spine: an MRI study. Eur Spine J - Offic Publ Eur Spine Soc Eur Spinal Deformity Soc Eur Section Cervical Spine Res Soc 1999 Oct;8(5): 396-401. 